\title{
REVIEW
}

\section{Spinal cord injury sequelae alter drug pharmacokinetics: an overview}

\author{
H Mestre, T Alkon, S Salazar and A Ibarra \\ Facultad de Ciencias de la Salud, Universidad Anáhuac México Norte, Huixquilucan Edo. de México, México
}

\begin{abstract}
Study design: Literature review.
Objectives: Critical review of the literature published on the physiological alterations caused by spinal cord injury $(\mathrm{SCl})$ and their effect on the pharmacokinetic parameters of commonly employed drugs. The review introduces the most recent treatment protocols of a variety of drugs, enabling the modern clinician to apply efficacious and cost-effective solutions to the pharmacological treatment of $\mathrm{SCl}$ patients.

Methods: Studies published in international indexed journals up to January 2011 were selected from the PubMed database.

Results: The review evaluated the sequelae of $\mathrm{SCl}$ and their effect on pharmacological processes. The results demonstrated that these alterations affected the pharmacokinetics of drugs commonly administered to $\mathrm{SCl}$ patients, such as antibiotics, muscle relaxants, immunosuppressants and analgesics. Conclusion: There are multiple etiologies to $\mathrm{SCl}$ and patients present with varying degrees of impairment. Factors such as level of injury and completeness of the injury create a very heterogeneous population within the $\mathrm{SCl}$ community. The heterogeneity of this population creates a problem when trying to standardize pharmacokinetic (PK) parameters. It is because of this that specific physiological alterations must be linked to changes in PK and be identified within the clinical setting. This relationship between physiology and PK enables the clinician to be alert for possible pharmacological complications in individual patients based on their clinical manifestations. Future research should aim to develop rigorous therapeutic guidelines tailored to the diverse manifestations of $\mathrm{SCl}$ so as to provide effective, affordable and safe pharmacotherapy.
\end{abstract}

Spinal Cord (2011) 49, 955-960; doi:10.1038/sc.2011.58; published online 31 May 2011

Keywords: autonomic dysreflexia; pharmacokinetics; paraplegia; pathophysiology; pharmacotherapy

\section{Introduction}

After spinal cord injury (SCI), a series of degenerative phenomena alter metabolic and physiological functions. These alterations persist throughout the acute, subacute and, in most cases, chronic phases of disease. A SCI isolates the uninjured spinal cord below the injury site, from the brain and the rest of the central nervous system. ${ }^{1}$ This mechanism of decentralization impairs communication between the central nervous system and the peripheral nervous system, resulting in an autonomic nervous system failure. SC-injured individuals may develop spinal shock and autonomic dysreflexia as a result of these permanent disturbances in global homeostasis. ${ }^{2}$ These life-long alterations convert SCI patients into a heterogeneous population with a physiology different from that of healthy individuals.

Correspondence: Professor A Ibarra, Facultad de Ciencias de la Salud, Universidad Anáhuac México Norte, Av. Universidad Anáhuac No. 46, Col. Lomas Anáhuac, C.P. 52786, Huixquilucan Edo. de México, México.

E-mail: iantonio65@yahoo.com or jose.ibarra@anahuac.mx

Received 26 September 2010; revised 19 April 2011; accepted 21 April 2011; published online 31 May 2011
Currently, the incidence of SCI is between 12.1-57.8 new cases per million inhabitants every year worldwide. ${ }^{3}$ In the past, acute-stage mortality reduced the prevalence of SCI; however, because of recent scientific advancements, patients now have a 47-51-year life expectancy. ${ }^{4}$ In spite of the improved life expectancy, SCI patients suffer from other challenges, such as severe financial, social and psychological burdens.

One of the major sources of financial burden is drug therapy. Injured patients develop secondary complications that require large quantities of medication generating an annual cost of $\$ 2489$ USD. $^{5}$ Research has proven that physiological and metabolic functions are altered in people with SCI; therefore, the fundamental assumptions of pharmacokinetics (PK) and pharmacodynamics derived from able-bodied individuals do not apply. In many cases, the standard schedule of the therapy does not achieve the desired therapeutic effects or entails drug concentrations at potentially toxic levels for the patient. ${ }^{6}$ Therefore, there is significant need for accurate and precise pharmacological 
schedules specifically developed for this population. It is imperative to modify the current treatment protocols for a population-specific approach to pharmacology that optimizes the absorption, distribution, metabolism and excretion of drugs in SCI patients. This manuscript reviews the main physiological and metabolic alterations in SCI patients and their effect on PK parameters and introduces the most recent population-specific treatment protocols of a variety of drugs, enabling the modern clinician to apply efficacious and cost-effective solutions to the pharmacological treatment of SCI patients.

The length of the spinal cord and the distribution of the functional ascending and descending tracts make location of the lesion very important in determining the clinical manifestations of SCI. These factors make the SCI community highly heterogeneous and therefore complicate the development of standard PK models. It is the clinician's responsibility to understand the sequelae that their SCI patient possesses and determine the possible alterations in PK that may cause complications in traditional drug therapy.

\section{Methods}

Review of the literature consisted of a methodological search on the PubMed database. The primary search used keywords such as SCI therapy, SCI PK, SCI altered PK, SCI PK absorption, SCI PK distribution, SCI PK metabolism and SCI PK excretion. Studies relevant to the review were taken into consideration. The last literature review published on this topic (1989) was also incorporated into this review. Only studies that had been published in international indexed journals up to January 2011 were used. The results were included if the studies achieved a significant statistical difference $(P<0.05)$ against controls. Studies that satisfied the criteria were analyzed and reported. Analysis consisted of identifying the PK parameter that is altered in SCI and the process causing it. The secondary search used the following keywords: SCI pathophysiology, SCI sequelae, SCI altered physiology, SCI syndrome, spinal shock, neurogenic shock, spasticity and autonomic dysreflexia. Owing to the progressive understanding of SCI pathophysiology, only studies published in indexed journals after 2007 were taken into consideration.

\section{Results}

The importance of understanding the altered physiology and its implications on PK warrants a brief review of the pathophysiology and sequelae subsections. The goal is to help physicians understand which patients may present altered PK based on their clinical manifestations. The subsections were organized so that readers can further understand the alterations in PK and their causes.

\section{Sequelae}

Neuromuscular. Immediately after SCI, the patient succumbs to spinal shock syndrome. This consists of a state of areflexia and flaccid paralysis. As the patient enters the subacute phase of SCI, spinal shock resolves and paralysis of the limbs below the lesion occurs owing to the conductive impairment of the descending tracts. This phase is no longer characterized by flaccid paralysis but rather by a state of spastic paralysis. The spastic muscle groups will depend on which tracts are affected by the SCI. ${ }^{7}$ Paralysis leads to a loss in neural tone causing a marked increase in muscle wasting. ${ }^{8}$ If there is muscular atrophy, it reduces the biomass that a drug must cross to enter systemic blood flow and may therefore pose alterations in IM absorption of certain drugs.

On the other hand, if descending visceral autonomic fibers are also interrupted, bladder and intestinal functions will no longer be under functional control; the implications of these will be discussed later.

Cardiovascular. If the injury to the spinal cord is above the level of the sixth thoracic $\left(\mathrm{T}_{6}\right)$ vertebrae, this will affect sympathetic innervation of the cardiovascular system and the autonomic nervous system becomes unbalanced. ${ }^{9}$ The decrease in sympathetic tonus promotes vasodilatation, resulting in hypotensive crisis, hypoperfusion of vital organs and hypoxemia. Eventually, partial autonomic regulation returns and can trigger autonomic dysreflexia. Sensorial stimuli from peripheral nerves are prevented from reaching the inhibitory supraspinal centers, causing the sympathetic ganglia to become hyperexcitable. ${ }^{9}$ Sympathetic hyperactivity causes increased vasoconstriction below the injured spinal cord. ${ }^{2}$ Vasoconstriction induces an elevated BP, and thereby the central nervous system tries to lower BP by activating injury-excluded parasympathetic stimulation of the heart, which triggers a state of bradycardia and vasodilation above the site of injury. ${ }^{10}$ The hemodynamic instability alters normal blood flow. Bradycardic hypotension results in hypoperfusion, which can have a negative effect on drugs administered through intravenous or intraarterial routes and could decrease microvascular blood flow to certain organs. Studies show that SCI induces a significant decrease in microvascular blood flow in the liver, the spleen, muscle and fore footpad skin in rats. ${ }^{11}$ These animal models allow us to extrapolate a similar pathophysiology in SC-injured humans. These alterations in blood flow and perfusion may affect the distribution of some drugs.

Respiratory. The loss of supraspinal regulatory mechanisms over the respiratory system results in the acute loss of motor function of the respiratory muscles and complications such as respiratory failure, atelectasis, neurogenic pulmonary edema and pneumonia. ${ }^{12}$ Augmented breaths, the cough reflex and expiration reflexes can be regained after SCI due to plasticity of premotor neurons in the thoracic spinal cord. ${ }^{13}$ The limited or complete loss of somatic pulmonary control makes the proper absorption of inhaled drugs difficult.

Gastrointestinal. Depending on the level of injury, the digestive tract of the SCI patient could develop physiological alterations in gastric emptying, intestinal motility and dysreflexia of both the colo-gastric and recto-anal reflexes. Dyspepsia is a common complication in SCI patients because 
of the interrupted innervation of supraspinal centers to the enteric nervous system. There is a delayed gastric emptying in this patients that could be due to the activation of the colo-gastric inhibitory reflex triggered by inappropriate colonic emptying. ${ }^{14,15}$ The cause of slowed colonic emptying was discovered to be rooted in the autonomic innervations of the hindgut. Owing to the particular innervations of the hindgut, this segment of the colon becomes isolated from supraspinal control immediately after SCI. ${ }^{16}$ The deinnervated hindgut decreases colonic motility and triggers the colo-gastric inhibitory reflex, slowing overall gastrointestinal (GI) motility. ${ }^{15}$ The summation of all of these altered processes results in the SCI-characteristic syndrome known as spastic neurogenic bowel. ${ }^{17}$ Spastic neurogenic bowel requires the frequent use of laxatives in SCI patients; unfortunately, there are no population-specific PK studies of commonly used laxatives. Slowed gastric emptying and decreased intestinal motility are one of the most consequential sequelae in PK alterations post-SCI. This is because they alter the absorption of per oral route drugs. Altered GI absorption in SCI patients is also affected by the physicochemical properties of each drug; this will be discussed further in the coming sections.

Urinary. After SCI the genitourinary system is not spared, and posterior to autonomic dysfunction an array of alterations begins to develop. The most important fraction of the genitourinary system is the kidney, which solely depends on spinal cord-mediated innervations. The autonomic innervation of the kidney is entirely sympathetic, as they lack parasympathetic nerve fibers. SCI doubles renal sympathetic activity, altering renal arterial blood flow, reducing sodium and potassium excretion, and lowering renal plasma flow dramatically. ${ }^{18}$ Altered electrolyte excretion leads to the formation of renal calculi, which exacerbate the altered physiology of the kidney. ${ }^{19}$ Decrease in urinary excretion causes drug molecules to remain in systemic blood flow for longer periods of time, increasing their half-life. Longer halflives mean that longer dosing intervals are needed to prevent the drug concentration from reaching toxic levels. Following SCI, the bladder is afflicted by detrusor hyperreflexia and increased activity of the urethral sphincter. $^{20,21}$ Bladder detrustor and external urethral sphincter dyssynergia renders micturition impossible for SCI patients, ${ }^{22}$ therefore necessitating Foley catheterization. Chronic catheterization in SCI patients often results in urinary tract infections and vesicoureteral reflux that may lead to pyelonephritis. Chronic catheterization results in the need for prophylactic antibiotics, such as aminoglycosides. Amikacin, an aminoglycoside, depends on urinary excretion and therefore has an increased half-life in SCI patients. When administered under normal dosing intervals, amikacin may cause toxicity in SCI individuals.

Integumentary. Autonomic dysfunction on a systemic scale disrupts homeostatic blood flow and may lead to defective microvascular circulation of the skin. Collagen degradation $^{23,24}$ and limited microvascular blood flow to the skin could result in alterations in the absorption of drugs requiring the transdermal route.

\section{Altered PK}

To better understand the variations that exist in PK they are being analyzed according to absorption, distribution, metabolism (biotransformation) and excretion (elimination).

Absorption. Analysis of the advantages and disadvantages of the administration routes of drugs to the SCI population is crucial. The administration route is determined by the various characteristics of the drug. Oral ingestion is the most common method of administration, because it is safe, convenient and cost-effective owing to the patient's ability to self-administer the drug without the need of professional assistance. In spite of this, the oral route depends on GI absorption of the drug, which may pose an array of disadvantages. The efficient absorption of drugs via the GI tract depends on several factors, including drug solubility in water, tissular blood flow and the specific site of the GI tract where absorption will occur. In SC-injured patients, the oral route may be disadvantageous owing to impaired GI motility. Because of slowed gastric, intestinal and colonic peristalsis, the rate of drug transport to its site of absorption and rate of absorption will be diminished. The chemical properties of specific drugs determine the speed of absorption. Absorption in the GI tract depends on passive diffusion and, therefore, is favored when the drug is in a non-ionized lipid-soluble form. This seemingly simplistic process is complicated by an additional factor: the fluctuating GI tract $\mathrm{pH}$. The ability of drugs to diffuse across the lipid mucosal barrier of the stomach and intestine depends on their $\mathrm{pK}_{\mathrm{a}}$. Weak acids are not in a protonated form in acidic environments (for example, the stomach) and will readily diffuse. Weak bases ionize in the stomach, preventing its absorption, but do not ionize in a basic $\mathrm{pH}$ environment (for example, intestine). Thus, in SCI individuals, absorption of basic drugs is prolonged, as slowed gastric emptying retards content mobilization toward the alkaline environment of the intestines. In SCI patients, oral-administrated drugs such as aspirin and paracetamol (weak acids and analgesic drugs) take longer to reach the maximum concentration in plasma compared with non-injured controls. ${ }^{25}$ This phenomenon is attributed to the effect of slowed gastric emptying and GI motility because the drugs take longer to reach its main absorption site (small intestine). As a consequence of delayed absorption, the time span for peaking plasma concentrations achieving the therapeutic effect of these drugs is increased. Other drugs commonly prescribed to the SCI patient that have shown to be affected by delayed gastric emptying and decreased GI motility are theophylline, ${ }^{26}$ dantrolene, ${ }^{27}$ carbamazepine, $^{28}$ 4-aminopyridine, ${ }^{29}$ cyclosporin $\mathrm{A}^{30}$ and baclofen. ${ }^{31}$ Theophylline is a methylxanthine drug used for the treatment of bradycardia ${ }^{32}$ or to promote the recovery of the hemidiaphragmatic function of SCI individuals. ${ }^{33}$ The muscle relaxant Dantrolene is used to reduce symptoms of spasticity such as hyperreflexia, increases in muscle tone and involuntary muscle activity, as well as in chronic pain; it may 
also exert a role as neuroprotective agent after SC injury. ${ }^{34}$ Another drug extensively used to reduce chronic neuropathic pain is the anticonvulsant carbamazepine, ${ }^{35}$ which has been shown to have neuroprotective effects after SCI. ${ }^{36}$ Fampridine, also known as 4-aminopyridine, is a potassium channel blocker that significantly restores axonal conduction in the injured spinal cord. ${ }^{37}$ Cyclosporin-A is an immunosuppressant agent that has recently been demonstrated to have neuroprotective and neuroregenerative effects in SCI models. ${ }^{38}$ Finally, baclofen is a centrally acting muscle relaxant used to treat spasticity. ${ }^{31}$

The absorption of these drugs is significantly reduced when administered orally. ${ }^{25-31}$ Shown to be absorbed in the intestines, all of these drugs exhibit differences in plasma concentration levels compared with gastric absorption drugs. The solution to altered PK of orally disposed drugs could be complementary dosing with metoclopramide, a gastroprokinetic. $^{39}$ The use of metoclopramide at an initial oral dose of $10 \mathrm{mg}, 30 \mathrm{~min}$ before ingestion of the desired drug, improves its absorption. ${ }^{27}$

Other routes of administration pose limitations for the physiology of SCI patients. This review previously stated that altered venous blood flow and limited microvasculature irrigation to the dermis and musculature is present in these patients. Because of these altered processes, administration routes such as sublingual and transdermal application may present anomalous absorption; nonetheless, research regarding these topics is lacking. Studies on intramuscular administration of cefotiam and gentamicin have confirmed that both present delayed absorption and lower peak serum concentrations. ${ }^{40}$ Absorption of diclofenac through intramuscular administration, however, was unaltered in SCI patients. ${ }^{41}$ Further studies are needed to understand the distinct behavior of drugs and the physiological anomalies that cause PK alteration after intramuscular administration. Nevertheless, PK alterations should be closely attributed to the diverse chemical structure of drugs as well as to the loss of muscle mass, which decreases the biomass that drugs must travel through to reach major vasculature.

The inability of patients to control somatic inspirational mechanisms in high-level cervical injury can present obstacles in respiratory absorption; similarly, dyssynergia of the respiratory accessory muscles predisposes the patient to poor pulmonary function, rendering the pulmonary route of drug administration inefficient. The alterations in collagen metabolism present in SCI patients may alter standard PK values for transdermal absorption. As there have been no studies on the latter, more research should be conducted.

In view of the aforementioned alterations in PK in SCI patients and the lack of population-specific therapeutic protocols, physicians should rely on the intravenous administration of drugs whenever possible, under the treatment circumstances.

Distribution. Depending on drug bioavailability, only some drugs ultimately reach the target tissues after absorption. Once a drug enters systemic blood flow, the molecules must diffuse toward the target tissue, where they exert their effect.
In the human body, the fastest and most efficient way to distribute compounds into the interstitial and intracellular fluids is through systemic blood flow. A number of physiological factors affect and control this mechanism: cardiac output, regional blood flow, capillary permeability and tissue volume determine the rate of delivery and quantity of distribution into tissues. Greater blood flow to particular organs such as the liver and kidneys dictates that these areas will receive most of the drug, whereas muscle, skin, viscera and fat will receive it at shorter intervals and in smaller quantity. The distribution of drugs into target tissue depends on a physicochemical process known as bloodtissue partitioning. These mechanisms occur because of the drugs' ability to bind with plasma proteins and tissue macromolecules. ${ }^{42}$ In relation to SCI, several alterations in blood plasma constituents become problematic for drug distribution. Albumin is the main plasma protein and is responsible for carrying drugs through systemic blood flow; however, SCI patients commonly present hypoalbuminemia. This pathology underlies the altered distribution and PK in this subpopulation. Population-specific alterations in drug distribution kinetics are not available, but a small array of drugs has been found to have irregular distribution in SCI patients. Owing to low albumin levels, drugs with high plasma protein binding such as naproxen (99\% plasma protein binding) will have increased distributions, whereas those with low plasma protein binding, such as phenacetin (30\%), gentamicin and amikacin (both $10-20 \%),{ }^{43}$ will have normal distribution. After SCI there is an alteration in IL-2 expression, ${ }^{44}$ which leads to a redistribution of albumin-rich intravascular fluid to extravascular sites. ${ }^{45}$ In hydrophilic aminoglycosides such as gentamicin and amikacin, this will severely affect the volume of distribution. Studies have shown that increases in the volume of distribution post-SCI can range from $20 \%$ for amikacin to $70 \%$ in cases such as cefotiam. ${ }^{40}$ Other drugs with altered distribution due to an increase in volume of distribution include ketamine and lorazepam. ${ }^{46,47}$ Moreover, altered serum lipid profile affects the quantity of drug that can bind and be distributed.

Metabolism. Also known as biotransformation, drug metabolism takes place mainly in the cytochrome P450 (CYP) complex of the hepatocyte smooth endoplasmic reticulum. The cytochrome P450 superfamily isozymes are responsible for hepatic oxidation of drug molecules, which requires adequate hepatic blood flow. After SCI, hepatic microvascular blood flow is decreased, and hepatocyte gene expression and cathecolamine metabolism are affected. ${ }^{48}$ The precise etiology of how these alterations cause deviations from standard PK metabolism is unknown. These physiological alterations pose a disadvantageous outcome for high hepatic extraction drugs such as phenacetin, methylprednisolone and cyclosporin A. These drugs present an increased bioavailability due to impaired hepatic metabolic clearance. $^{30,43,49}$ Experimental studies have shown that increasing hepatic microvascular blood flow through nitric oxide-mediated vasodilation using L-arginine, a nitric oxide precursor, could ameliorate PK alterations at this level. ${ }^{50}$ 
This alteration only applies to drugs that require hepatic oxidation (for example, diazepam), because normal metabolic PK is seen in drugs that depend on conjugation in the liver. ${ }^{40}$ Compared with other PK parameters, metabolic alterations are minimal in SCI patients; nonetheless, changes in dosage quantity and intervals must be placed under scrutiny to optimize these therapies.

Elimination. Several pathways provide for drug molecule elimination. The integrity of drugs upon excretion depends on their chemical structure. Some drugs, such as gentamicin, are polar and can therefore be eliminated in the form they were absorbed; others are converted into polar metabolites. The renal pathway of excretion is the most important, and depends on glomerular filtration, active tubular secretion and passive tubular reabsorption. Minor fluctuations in normal renal function take place on a daily basis, and a constant $1 \%$ loss per year has a significant effect on drug elimination. ${ }^{42}$ The smallest decrease in renal function, as seen in the case of SCI, can develop into drug toxicity. Other excretion pathways are bile, feces, sweat, saliva and tears. Altered physiology in SCI patients prolongs the half-life of most drugs because of slowed renal clearance. Previously, it was stated that the kidney is innervated solely by sympathetic nerve fibers, which control glomerular filtration and other crucial processes of excretion. Overall studies on SCI patients have concluded that this population suffers from decreased renal function. The effect of this altered physiology decreases clearance and elevates the elimination half-life of the drug; examples of this phenomenon have been seen in amikacin, cefotiam, doxycycline, ketamine, diclofenac, vancomycin and lorazepam. ${ }^{41,46,51,40,47,52}$ In view of the above observations, patients may require longer dosing intervals to prevent drug toxicity. Reports have also suggested that if ibuprofen drug therapy is initiated, and the patient develops signs of drug toxicity, this may be a sign of ibuprofen-induced renal insufficiency, which further lowers drug clearance. ${ }^{53}$ On the other hand, drugs such as gentamicin do not produce an elevated elimination half-life and may be administered with standardized dosing intervals, taking into consideration alterations in the other PK criteria. In patients with renal insufficiency, alternative drugs with non-renal excretion and normal absorption include ciprofloxacin for antibiotic prophylaxis. ${ }^{54}$ Renal function is paramount when considering PK; organ function tests, such as creatinine excretion, should be performed whenever possible.

\section{Conclusion}

SCI poses a vast variety of challenges for the modern clinician; however, efficacious and cost-effective pharmacological therapeutics are feasible. There is significant need for accurate and precise administration schemes specifically developed for the heterogeneity of this population. Research has proven that physiological functions are altered in people with SCI, and therefore fundamental assumptions of PK based on non-SCI patients do not apply. It is imperative to modify the current treatment protocols for a populationspecific approach to pharmacology, one that optimizes the absorption, distribution, metabolism and excretion of drugs in SCI patients. The authors encourage the SCI scientific community to further research population-specific PK to optimize treatment in the clinical setting. Conducting this research would have a dramatic effect on patients with SCI, improving life quality and increasing life expectancy.

\section{Conflict of interest}

The authors declare no conflict of interest.

\section{References}

1 Privat A. Pathophysiology and treatment of spinal cord injury. Bull Acad Natl Med 2005; 189: 1109-1117.

2 Bravo G, Guizar-Sahagun G, Ibarra A, Centurion D, Villalon CM. Cardiovascular alterations after spinal cord injury: an overview. Curr Med Chem Cardiovasc Hematol Agents 2004; 2: 133-148.

3 van den Berg ME, Castellote JM, Mahillo-Fernandez I, de PedroCuesta J. Incidence of spinal cord injury worldwide: a systematic review. Neuroepidemiology 2010; 34: 184-192.

4 Shavelle RM, DeVivo MJ, Paculdo DR, Vogel LC, Strauss DJ. Longterm survival after childhood spinal cord injury. J Spinal Cord Med 2007; 30(Suppl 1): S48-S54.

5 Berkowitz M. The costs of spinal cord injury. In: Lin VW, Cardenas DD, Cutter NC, et al. (eds). Spinal Cord Medicine: Principles and Practice. Demos Medical Publishing: New York, 2003.

6 Segal JL, Brunnemann SR, Gray DR. Gentamicin bioavailability and single-dose pharmacokinetics in spinal cord injury. Drug Intell Clin Pharm 1988; 22: 461-465.

7 Brown P. Pathophysiology of spasticity. I Neurol Neurosurg Psychiatry 1994; 57: 773-777.

8 Qin W, Bauman WA, Cardozo C. Bone and muscle loss after spinal cord injury: organ interactions. Ann N Y Acad Sci 2010; 1211: 66-84.

9 Mathias CJ, Naik RB, Warren DJ, Frankel HL. Autonomic neuropathy. Arch Intern Med 1983; 143: 1635.

10 Garstang SV, Miller-Smith SA. Autonomic nervous system dysfunction after spinal cord injury. Phys Med Rehabil Clin N Am 2007; 18: 275-296.

11 Guizar-Sahagun G, Velasco-Hernandez L, Martinez-Cruz A, Castaneda-Hernandez G, Bravo G, Rojas G et al. Systemic microcirculation after complete high and low thoracic spinal cord section in rats. J Neurotrauma 2004; 21: 1614-1623.

12 Stevens RD, Bhardwaj A, Kirsch JR, Mirski MA. Critical care and perioperative management in traumatic spinal cord injury. J Neurosurg Anesthesiol 2003; 15: 215-229.

13 Bolser DC, Jefferson SC, Rose MJ, Tester NJ, Reier PJ, Fuller DD et al. Recovery of airway protective behaviors after spinal cord injury. Respir Physiol Neurobiol 2009; 169: 150-156.

$14 \mathrm{Lu}$ CL, Montgomery P, Zou X, Orr WC, Chen JD. Gastric myoelectrical activity in patients with cervical spinal cord injury. Am J Gastroenterol 1998; 93: 2391-2396.

15 Enck P, Klosterhalfen S. Placebo response in functional bowel disorders. Z Gastroenterol 2006; 44: 257-266.

16 Longo WE, Ballantyne GH, Modlin IM. The colon, anorectum, and spinal cord patient. A review of the functional alterations of the denervated hindgut. Dis Colon Rectum 1989; 32: 261-267.

17 Preziosi G, Emmanuel A. Neurogenic bowel dysfunction: pathophysiology, clinical manifestations and treatment. Expert Rev Gastroenterol Hepatol 2009; 3: 417-423.

18 Silver JR, Doggart JR. Reduced sodium output following acute spinal injury. Spinal Cord 2004; 42: 191-198.

19 Chen Y, DeVivo MJ, Stover SL, Lloyd LK. Recurrent kidney stone: a 25-year follow-up study in persons with spinal cord injury. Urology 2002; 60: 228-232. 
20 Birder LA, de Groat WC. Mechanisms of disease: involvement of the urothelium in bladder dysfunction. Nat Clin Pract Urol 2007; 4: $46-54$.

21 Smith CP, Gangitano DA, Munoz A, Salas NA, Boone TB, Aoki KR et al. Botulinum toxin type A normalizes alterations in urothelial ATP and NO release induced by chronic spinal cord injury. Neurochem Int 2008; 52: 1068-1075.

22 de Groat WC, Kawatani M, Hisamitsu T, Cheng CL, Ma CP, Thor K et al. Mechanisms underlying the recovery of urinary bladder function following spinal cord injury. J Auton Nerv Syst 1990; 30(Suppl): S71-S77.

23 Rodriguez GP, Claus-Walker J, Kent MC, Garza HM. Collagen metabolite excretion as a predictor of bone- and skin-related complications in spinal cord injury. Arch Phys Med Rehabil 1989; 70: 442-444.

24 Rodriguez GP, Claus-Walker J, Kent MC, Stal S. Adrenergic receptors in insensitive skin of spinal cord injured patients. Arch Phys Med Rehabil 1986; 67: 177-180.

25 Fuentes-Lara G, Guizar-Sahagun G, Garcia-Lopez P. Effect of experimental spinal cord injury on salicylate bioavailability after oral aspirin administration. J Pharmacol Toxicol Methods 1999; 42: 93-97.

26 Segal JL, Brunnemann SR, Gordon SK, Eltorai IM. The absolute bioavailability of oral theophylline in patients with spinal cord injury. Pharmacotherapy 1986; 6: 26-29.

27 Gilman TM, Segal JL, Brunnemann SR. Metoclopramide increases the bioavailability of dantrolene in spinal cord injury. J Clin Pharmacol 1996; 36: 64-71.

28 Reihani-Kermani H, Ansari M, Karamousian S. The influence of experimental spinal cord injury on carbamazepine pharmacokinetics. Arch Iran Med 2006; 9: 231-235.

29 Hayes KC, Potter PJ, Hsieh JT, Katz MA, Blight AR, Cohen R. Pharmacokinetics and safety of multiple oral doses of sustainedrelease 4-aminopyridine (Fampridine-SR) in subjects with chronic, incomplete spinal cord injury. Arch Phys Med Rehabil 2004; 85: 29-34.

30 Ibarra A, Guizar-Sahagun G, Correa D, Kretschmer R, Grijalva I, Flores-Murrieta FJ et al. Alteration of cyclosporin-A pharmacokinetics after experimental spinal cord injury. J Neurotrauma 1996; 13: 267-272.

31 Aisen ML, Dietz MA, Rossi P, Cedarbaum JM, Kutt H. Clinical and pharmacokinetic aspects of high dose oral baclofen therapy. J Am Paraplegia Soc 1992; 15: 211-216.

32 Schulz-Stubner S. The use of small-dose theophylline for the treatment of bradycardia in patients with spinal cord injury. Anesth Analg 2005; 101: 1809-1811.

33 Nantwi KD, Goshgarian HG. Effects of chronic systemic theophylline injections on recovery of hemidiaphragmatic function after cervical spinal cord injury in adult rats. Brain Res 1998; 789: 126-129.

34 Aslan A, Cemek M, Buyukokuroglu ME, Altunbas K, Bas O, Yurumez Y et al. Dantrolene can reduce secondary damage after spinal cord injury. Eur Spine J 2009; 18: 1442-1451.

35 Finnerup NB, Gottrup H, Jensen TS. Anticonvulsants in central pain. Expert Opin Pharmacother 2002; 3: 1411-1420.

36 Sirlak M, Eryilmaz S, Bahadir IM, Sirin YS, Besalti O, Yazicioglu L et al. Effects of carbamazepine on spinal cord ischemia. J Thorac Cardiovasc Surg 2008; 136: 1038-1043.
37 Cardenas DD, Ditunno J, Graziani V, Jackson AB, Lammertse D, Potter $\mathrm{P}$ et al. Phase 2 trial of sustained-release fampridine in chronic spinal cord injury. Spinal Cord 2007; 45: 158-168.

38 Ibarra A, Diaz-Ruiz A. Protective effect of cyclosporin-A in spinal cord injury: an overview. Curr Med Chem 2006; 13: 2703-2710.

39 Nimmo J, Heading RC, Tothill P, Prescott LF. Pharmacological modification of gastric emptying: effects of propantheline and metoclopromide on paracetamol absorption. $\mathrm{Br}$ Med J 1973; 1: 587-589.

40 Segal JL, Brunnemann SR. Clinical pharmacokinetics in patients with spinal cord injuries. Clin Pharmacokinet 1989; 17: 109-129.

41 Garcia-Lopez P, Salas R. Bioavailability of diclofenac after intramuscular administration to rats with experimental spinal cord injury. J Pharmacol Toxicol Methods 1999; 42: 99-101.

42 Buxton, ILO. Pharmacokinetics and pharmacodynamics: the dynamics of drug absorption, distribution, action, and elimination. In: Brunton LL, Lazo JS and Parker KL (eds) Goodman \& Gilmańs The Pharmacological Basis of Therapeutics, 11th edn. MacGraw-Hill: Iowa, 2006. http://www.accessmedicine.com/ features.aspx.

43 Garcia-Lopez P, Martinez-Cruz A, Guizar-Sahagun G, CastanedaHernandez G. Acute spinal cord injury changes the disposition of some, but not all drugs given intravenously. Spinal Cord 2007; 45: 603-608.

44 Segal JL, Gonzales E, Yousefi S, Jamshidipour L, Brunnemann SR. Circulating levels of IL-2R, ICAM-1, and IL-6 in spinal cord injuries. Arch Phys Med Rehabil 1997; 78: 44-47.

45 Katz J, Bonorris G, Sellers AL. Extravascular albumin in human tissues. Clin Sci 1970; 39: 725-729.

46 Hijazi Y, Bodonian C, Bolon M, Salord F, Boulieu R. Pharmacokinetics and haemodynamics of ketamine in intensive care patients with brain or spinal cord injury. Br J Anaesth 2003; 90: 155-160.

47 Segal JL, Brunnemann SR, Eltorai IM, Vulpe M. Decreased systemic clearance of lorazepam in humans with spinal cord injury. J Clin Pharmacol 1991; 31: 651-656.

48 Segal, JL, Brunnemann, SR, Castañeda-Hernández, G, GuizarSahagún, G. Altered hepatocyte gene expression in a rat model of chronic spinal cord injury. J Clin Pharmacol 2000; 40: 1045-1065.

49 Segal JL, Maltby BF, Langdorf MI, Jacobson R, Brunnemann SR, Jusko WJ. Methylprednisolone disposition kinetics in patients with acute spinal cord injury. Pharmacotherapy 1998; 18: 16-22.

50 Vertiz-Hernandez A, Castaneda-Hernandez G, Martinez-Cruz A, Cruz-Antonio L, Grijalva I, Guizar-Sahagun G. L-arginine reverses alterations in drug disposition induced by spinal cord injury by increasing hepatic blood flow. J Neurotrauma 2007; 24: 1855-1862.

51 Lavezo LA, Davis RL. Vancomycin pharmacokinetics in spinal cord injured patients: a comparison with age-matched, ablebodied controls. J Spinal Cord Med 1995; 18: 233-235.

52 Brunnemann SR, Segal JL. Amikacin serum protein binding in spinal cord injury. Life Sci 1991; 49: L1-L5.

53 Dahlin PA, George J. Baclofen toxicity associated with declining renal clearance after ibuprofen. Drug Intell Clin Pharm 1984; 18: 805-808.

54 Pedersen SS, Hørbov S, Biering-Sørensen F, Høiby N. Peroral treatment with ciprofloxacin of patients with spinal cord lesion and bacteriuria caused by multiply resistant bacteria. Paraplegia 1990; 28 : 41-47. 